\title{
Gastrointestinal Symptoms in Elderly Hemodialysis Patients
}

\author{
Kaptanogullari $\mathrm{H}^{1}$ and Harmankaya $\mathbf{O}^{2 *}$ \\ ${ }^{1}$ Vocational School of Health Sciences, Biruni University, Turkey \\ ${ }^{2}$ Department of Internal Medicine, Biruni University, Turkey
}

*Corresponding author: Harmankaya 0, Department of Internal Medicine, Division of Nephrology, Faculty of Medicine, Biruni University, Turkey.

To Cite This Article: Kaptanogullari H, Harmankaya O. Gastrointestinal Symptoms in Elderly Hemodialysis Patients. Am J Biomed Sci \& Res. 2021 - 12(5). AJBSR.MS.ID.001786. DOI: 10.34297/AJBSR.2021.12.001786.

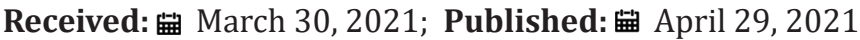

\begin{abstract}
Aim: Gastrointestinal symptoms are common in patients with end-stage renal disease undergoing renal replacement therapy. The aim of this cross-sectional study was to determine the prevalence and diversity of gastrointestinal symptoms in elderly hemodialysis patients.

Methods: Elderly patients (>75 years of age) of four outpatient hemodialysis service centers were given a questionnaire to complete which was previously validated and designed to measure the occurrence of gastrointestinal symptoms within the last six months. An interview with a nephrologist was completed at the same time.

Results: A total of 112 patients responded ( 67 females, 45 males) to the questionnaire. Our study revealed a high prevalence of gastrointestinal symptoms in elderly patients. Ninety-four percent of elderly dialysis patients had at least one of the following chronic gastrointestinal symptoms: dyspeptic symptoms (especially bloating) was reported in 53\%, chronic constipation was reported in $42 \%$, pyrosis in $25 \%$ and abdominal pain in $22 \%$ of the patients. Thirty-eight percent had loss of appetite and $14 \%$ had chronic diarrhoea. Patients were moderately/severely bothered by symptoms in up to $63 \%$ of cases. Nausea and vomiting were the least prevalent symptoms. There was no significant statistical difference between the severity of symptoms by gender ( $\mathrm{p}=0.483)$. Diabetes mellitus was not associated with more gastrointestinal symptoms ( $\mathrm{p}=0.662)$.

Conclusion: The prevalence of gastrointestinal symptoms is high in elderly dialysis patients and these symptoms cause a major health burden in their daily life. Our study showed no significant relationship between the severity of gastrointestinal symptoms and gender. Diabetic patients showed no substantial rise in the amount or severity of gastrointestinal symptoms as compared to non-diabetic patients.
\end{abstract}

Keywords: Gastrointestinal symptoms, Elderly patients, Hemodialysis therapy

\section{Introduction}

Gastrointestinal symptoms are widespread in patients undergoing maintenance hemodialysis and are a major cause of morbidity [1-5]. These symptoms are more common in older adults because of changes associated with aging, comorbidities such as diabetes, and medications they have to take. Delayed motility, decrease in gastric secretion, and decreased absorption in the elderly may be responsible for gastrointestinal symptoms $[1,6,7]$.

The increased concentration of gastrointestinal hormones, and changes of intestines due to uremia may be linked to gastrointestinal symptoms. Changes of diet, gastroparesis and prolonged gastric emptying also influence these symptoms in elderly adults $[8,9]$.

Old patients on hemodialysis have more comorbidities such as diabetes, heart disease and hypertension. Studies focusing on these patients showed more severe gastrointestinal symptoms compared to younger patients [3].

Our aim was to gather information on the prevalence of chronic gastrointestinal symptoms in elderly hemodialysis patients. We also assessed the relationship of gastrointestinal symptoms with gender and diabetes. 


\section{Methods}

We included all elderly hemodialysis patients older than 75 years who had been receiving dialysis for at least six months in four hemodialysis centers in Istanbul, Turkey. The severity of gastrointestinal symptoms was determined by a patient administered gastrointestinal symptoms questionnaire, which divided patients into three categories: mild, moderate, and severe. The study was conducted through a face-to-face interview with a nephrologist to ensure that patients understood the questions. Details on co-morbid conditions, medications and duration of dialysis were retrieved from medical notes. Patients diagnosed with a gastrointestinal disease, infectious illness, malignancies and who were hospitalized in the last 3 months were excluded.

\section{Statistical Analysis}

Data were coded and entered into the Statistical Package for Social Sciences program (SPSS) for analysis. Statistical analysis was performed with SPSS, version 23.0 (IBM, San Jose, CA, USA). Comparisons were made between female and male patients as well as diabetic and non-diabetic patients. Continuous variables were expressed as mean \pm standard deviation and categorical variables were presented as absolute (number of participants) and relative frequencies (percentages). Continuous variables were compared using either Student's t-test, whereas the categorical variables were compared with Pearson's $\chi 2$ analysis test where appropriate. A significant difference was considered when $\mathrm{p}<0.05$.

\section{Results}

Clinically stable geriatric hemodialysis patients (older than 75 years old) who were dialysis outpatients were included in this crosssectional study. Diabetes mellitus (43\%) and hypertension (37\%) were the two primary causes of ESRD. Characteristics of the study population are shown in Table 1. A total of 112 patients responded to the questionnaire (67 females and 45 males). A nephrologist conducted face-to- face interviews with patients. The median age of participants was 81 years ( $81 \pm 6$; range $75-94$ ). The prevalence of diabetes mellitus type 2 corresponds to $43 \%$ of the total patients. Time on dialysis was $51 \pm 38$ months. Fifty-four patients were using gastrointestinal system drugs (48\%). Average body mass index of the patients was 23.8 \pm 5.3 (ranging between 19.2-29.8).

Table 1: Clinical features of the study population.

\begin{tabular}{|c|c|}
\hline Patients (total) & $\mathbf{n = 1 1 2}$ \\
\hline Female & $67(59.8 \%)$ \\
\hline Male & $45(40.2 \%)$ \\
\hline Age (years) & $75-94(81 \pm 6)$ \\
\hline Time On Dialysis (months) & $51 \pm 38$ \\
\hline DM2 & $48(43 \%)$ \\
\hline GIS Drug use & $54(48 \%)$ \\
\hline BMI & $23.8 \pm 5.3$ \\
\hline
\end{tabular}

Ninety-four percent of the patients on hemodialysis reported at least one gastrointestinal symptom. The most frequent symptoms were bloating and constipation, while vomiting was the least prevalent (Figure 1).

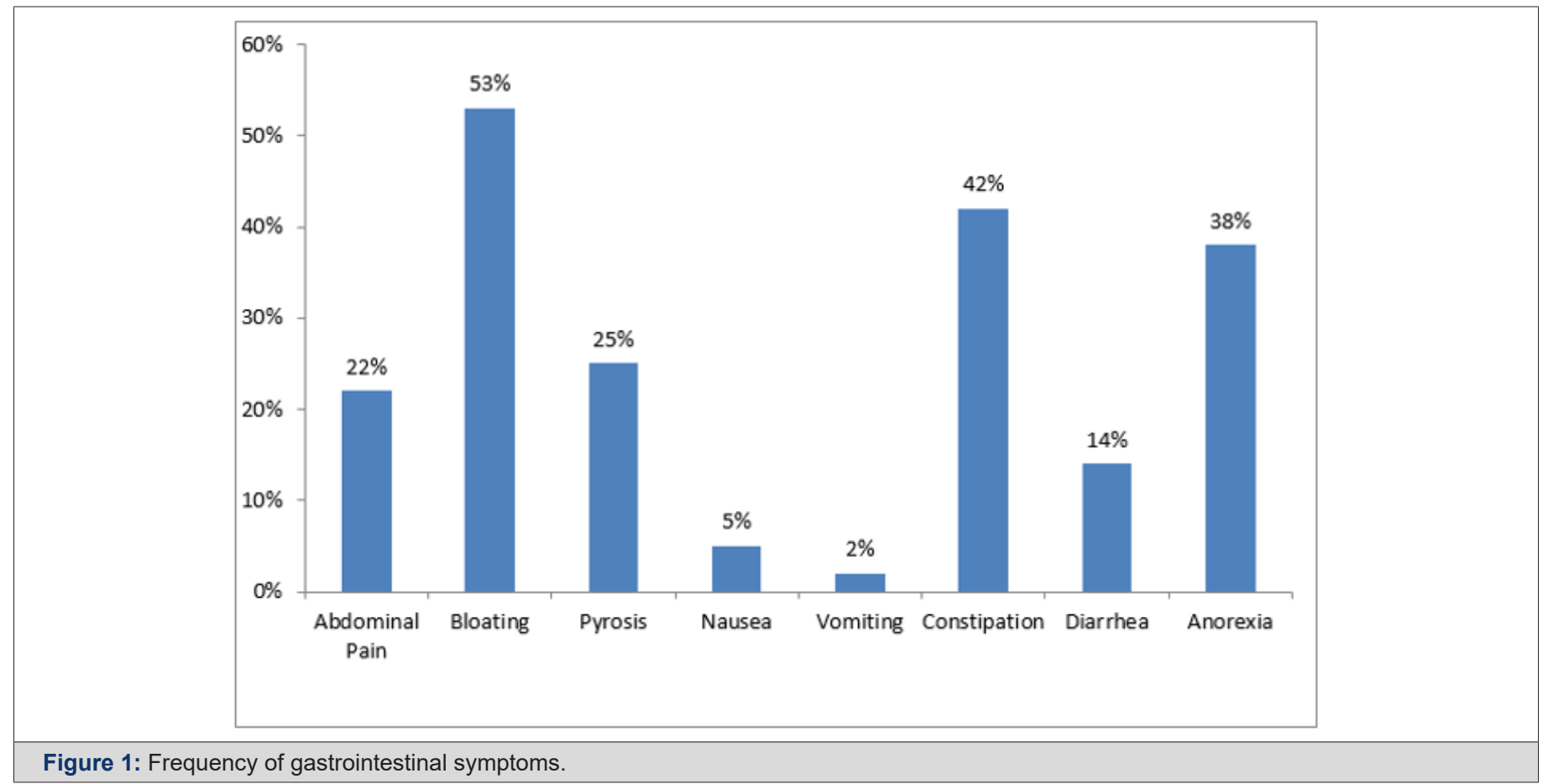


More than three gastrointestinal symptoms were reported by a substantially higher number of elderly hemodialysis patients.

Dyspeptic symptoms (especially abdominal bloating) were reported in 59 patients (53\%), chronic constipation was reported in 47 patients (42\%), pyrosis in 28 patients $(25 \%)$ and abdominal pain in 25 patients (22\%). Forty-three patients (38\%) had loss of appetite and 16 patients (14\%) had chronic diarrhoea. Nausea and vomiting were the least prevalent symptoms $(5 \%$ and $2 \%$, respectively) (Figure 1).

Three females and four males did not report any gastrointestinal symptom (6\%), 34 patients complained of mild symptoms (30\%), while 53 patients reported moderate symptoms (47\%) and 18 patients reported severe symptoms (16\%). Patients were moderately/severely bothered by symptoms in up to $63 \%$ of cases (Figure 2).

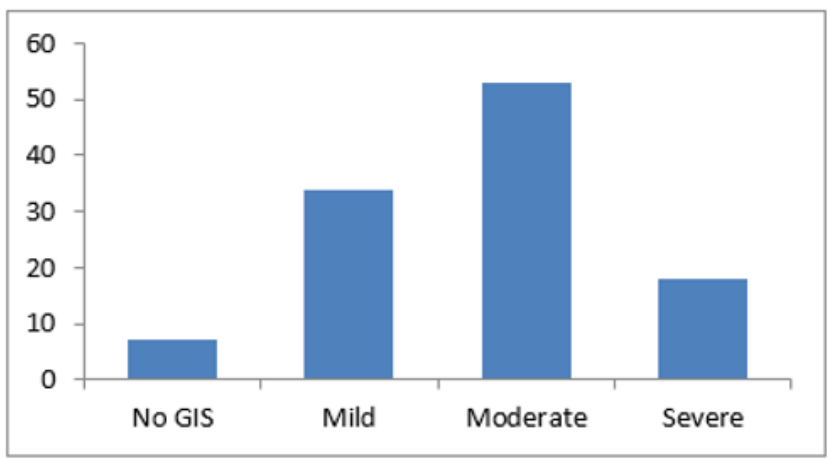

Figure 2: Intensity of gastrointestinal symptoms.

We did not find any association between the amount of gastrointestinal symptoms and gender $(p=0.64)$ (Table 2). There was no significant statistical difference between the severity of symptoms by gender ( $\mathrm{p}=0.483$ ) (Table 3 ).
Patients with diabetes showed no significant difference in the prevalence and severity of gastrointestinal symptoms compared to non-diabetics. Diabetes mellitus was not associated with more gastrointestinal symptoms ( $\mathrm{p}=0.73$ ) (Tables $4 \& 5$ ).

Table 2: Number of gastrointestinal symptoms by gender.

\begin{tabular}{|c|c|c|c|}
\hline & $\mathbf{0}$ & $\mathbf{1 - 3}$ & $\mathbf{\geq 4}$ \\
\hline Female & 3 & 42 & 22 \\
\hline Male & 4 & 27 & 14 \\
\hline
\end{tabular}

$p$ - value $=0.64$ (Pearson's $\times 2$ analysis)

Table 3: Intensity of gastrointestinal symptoms by gender.

\begin{tabular}{|c|c|c|c|c|c|}
\hline & Gender & $\mathbf{n}$ & Mean & Std. Deviation & Std. Error Mean \\
\hline \multirow{2}{*}{ Gastrointestinal Symptoms } & Female & 67 & 1.78 & 0.794 & 0.097 \\
\cline { 2 - 6 } & Male & 45 & 1.67 & 0.826 & 0.123 \\
\hline
\end{tabular}

p-value $=0.48$ (Student's $t$-test)

Table 4: Number of gastrointestinal symptoms by presence of diabetes.

\begin{tabular}{|c|c|c|c|}
\hline & $\mathbf{0}$ & $\mathbf{1 - 3}$ & $\mathbf{2}$ \\
\hline Diabetic & 2 & 33 & 13 \\
\hline Non-diabetic & 5 & 42 & 17 \\
\hline
\end{tabular}

$p$-value $=0.73$ (Pearson's $\times 2$ analysis)

Table 5: Intensity of gastrointestinal symptoms by presence of diabetes.

\begin{tabular}{|c|c|c|c|c|c|}
\hline & & n & Mean & Std. Deviation & Std. Error Mean \\
\hline \multirow{2}{*}{$\begin{array}{c}\text { Gastrointestinal } \\
\text { Symptoms }\end{array}$} & Non-diabetic & 64 & 1.7 & 0.77 & 0.096 \\
\cline { 2 - 6 } & Diabetic & 48 & 1.77 & 0.857 & 0.124 \\
\hline
\end{tabular}

$p$-value $=0.66$ (Student's $t$-test) 


\section{Discussion}

Gastrointestinal symptoms are very common in dialysis patients over the age of 75 years. There have been only a few clinical studies on these patients. Most of these studies have also found a high incidence of symptoms in elderly hemodialysis population $[6,10]$. Carrera-Jiménez et al. reported a very high prevalence of gastrointestinal symptoms $(90 \%)$ in geriatric patients on dialysis [10]. According to our results $94 \%$ of patients had gastrointestinal symptoms.

End-stage kidney disease is a difficult medical condition to manage, and gastrointestinal symptoms associated with uraemia, medications, and other comorbidities are common. Constipation, diarrhoea, bloating, abdominal pain, nausea, vomiting, and pyrosis have all been linked to a reduction in quality of life, as well as physical and mental well-being, and may contribute to reduced food intake, and the risk of malnutrition $[3,10]$.

Aging causes a decrease in gastrointestinal motility. Decrease in gastric secretions, decreased absorption and other alterations in gastrointestinal system functions have also been identified as part of aging. These changes are the underlying mechanisms that cause gastrointestinal symptoms like pyrosis, dyspepsia, constipation, loss of appetite, and malabsorption. They have a negative impact on the nutrition of geriatric patients and put them at risk of malnutrition $[6,10,11]$.

The causes of gastrointestinal symptoms may be iatrogenic (medications or strictly restrictive diets). Many medications used to treat chronic kidney disease such as phosphate binders, vitamin D analogues, erythropoietin-stimulating agents, and supplemental iron have important gastrointestinal side effects. Phosphate binders, for example, have been shown to increase the frequency of gastrointestinal symptoms such as constipation, bloating and abdominal pain $[12,13]$.

Our study revealed a high prevalence of gastrointestinal symptoms in geriatric patients, with abdominal bloating and constipation being more prevalent and severe. In our crosssectional study, the most common symptom experienced by elderly dialysis patients was abdominal bloating (53\%). Bossola et al. [2] also found a high incidence of bloating in dialysis patients. In a meta-analysis, the prevalence of bloating in hemodialysis patients ranged between 8-45.2\% [5]. Hemodialysis patients have to limit their intake of potassium-rich foods such as fruits, vegetables, and other fiber-rich foods, and constipation is a side effect of this kind of diet.

Yasuda et al. [14] showed that constipation was very common in hemodialysis patients compared to peritoneal dialysis patients. Fluid restriction, sedentary lifestyle and medications such as phosphate binders, may contribute to constipation in hemodialysis patients [1,3]. Zuvela et al. [5] investigated gastrointestinal symptoms in a total of 5161 dialysis (3804 HD and 1507 PD) patients across 30 studies and found that constipation was one of the most common gastrointestinal symptoms. Constipation was the second most common and severe presenting gastrointestinal symptom among elderly hemodialysis patients in our cross-sectional study.

Malnutrition is also an important criterion in the prognosis of patients with chronic renal failure and is associated with increased morbidity and mortality. In the elderly, malnutrition leads to additional decrease in quality of life $[10,15]$. Anorexia has been associated with malnutrition, poor quality of life, depression, greater hospitalization rates, and an increase in mortality [16]. Our study showed a high prevalence of anorexia in dialysis patients (38\%). Silva et al reported loss of appetite in $24.3 \%$ of 684 hemodialysis patients [17]. In a study of Bossola et al. [2] including 110 patients, the prevalence of anorexia was $27.3 \%$.

We found that $22 \%$ of patients reported abdominal pain associated with bloating. Hypotensive episodes, which are likely to occur during the early stages of dialysis, make elderly patients more susceptible to ischemia [18]. Therefore, abdominal pain may be observed more frequently in this group of patients.

Daniels et al reported that $90 \%$ of dialysis patients had at least one gastrointestinal symptom. Seventy percent had abdominal bloating and indigestion [19]. We found that 94\% of elderly hemodialysis patients had at least one chronic gastrointestinal symptom, whereas $53 \%$ experienced bloating.

Diabetes mellitus type 2 (DM2) is the main cause of chronic kidney disease. The presence of diabetes has been found to increase the number and severity of gastrointestinal symptoms [20]. In our study, patients with diabetes showed no significant difference in the prevalence and severity of gastrointestinal symptoms compared to non-diabetics. There have been other studies on this subject that reported similar findings $[1,7,10]$. Fiderkiewicz et al. [21] also did not observe any difference in the frequency of symptoms between diabetic and non-diabetic hemodialysis patients.

\section{Conclusions}

The current study showed that elderly patients on hemodialysis have a high prevalence of gastrointestinal symptoms, with abdominal bloating and constipation being more prevalent and severe. Further evaluation in a larger population of elderly dialysis patients is needed.

\section{Limitations}

The most important drawback of this study is that it is mostly observational. Another limitation was considering symptoms without evaluating factors like diet and medications closely. Future 
studies should focus on the type of diet and medications to better comprehend the effects of these symptoms on elderly dialysis patients.

\section{Acknowledgements}

The authors claim that the results presented in this paper have not been published previously in whole or part.

\section{References}

1. Cano AE, Neil AK, Kang JY, Barnabas A, Eastwood JB, et al. (2007) Gastrointestinal symptoms in patients with end-stage renal disease undergoing treatment by hemodialysis or peritoneal dialysis. Am J Gastroenterol 102(9): 1990-1997.

2. Bossola M, Luciani G, Rosa F, Tazza L (2011) Appetite and gastrointestinal symptoms in chronic hemodialysis patients. J Ren Nutr 21(6): 448-454.

3. Hans Strid, Magnus Simrén, Ann-Cathrine Johansson, Jan Svedlund, Ola Samuelsson, et al. (2002) The prevalence of gastrointestinal symptoms in patients with chronic renal failure is increased and associated with impaired psychological general well-being. Nephrol Dial Transplant 17(8): 1434-1439.

4. Thomas R, Panackal C, John M, Joshi H, Mathai S, et al. (2013) Gastrointestinal complications in patients with chronic kidney disease-a 5-year retrospective study from a tertiary referral center. Ren Fail 35(1): 49-55.

5. Zuvela J, Trimingham C, Le Leu R, l Faull R, Clayton P, et al. (2018) Gastrointestinal symptoms in patients receiving dialysis: A systematic review. Nephrology (Carlton) 23(8): 718-727.

6. Grassi M, Petraccia L, Mennuni G, Fontana M, Sarno A, et al. (2011) Changes, functional disorders, and diseases in the gastrointestinal tract of elderly. Nutr Hosp 26(4): 659-668.

7. Vui Heng Chong, Jackson Tan (2013) Prevalence of gastrointestinal and psychosomatic symptoms among Asian patients undergoing regular hemodialysis. Nephrology 18(2): 97-103.

8. Shirazian S, Radhakrishnan J (2010) Gastrointestinal Disorders and Renal Failure: Exploring the Connection. Nature Reviews Nephrology 6(8): 480-492.

9. Francois Folefack Kaze, Mathurin Kowo, Ethel Ngweh-Awah Anyu Ndikum, Hermine Danielle Fouda Menye, Victorine Nzana, et al. (2020) Prevalence and Determinants of Gastrointestinal Symptoms in Adults on Maintenance Hemodialysis in Cameroon. Open Journal of Nephrology 10(3): 212-222.
10. Carrera-Jiménez D, Miranda-Alatriste P, Atilano-Carsi X, Correa-Rotter R, Espinosa-Cuevas Á (2018) Relationship between Nutritional Status and Gastrointestinal Symptoms in Geriatric Patients with End-Stage Renal Disease on Dialysis. Nutrients 10(4): 425.

11. Davison SN, Jassal SV (2016) Supportive Care: Integration of PatientCentered Kidney Care to Manage Symptoms and Geriatric Syndromes. Clin J Am Soc Nephrol 11(10): 1882-1891.

12. Biruete A, Hill Gallant KM, Lindemann SR, Wiese GN, Chen NX, et al. (2020) Phosphate Binders and Nonphosphate Effects in the Gastrointestinal Tract. J Ren Nutr 30(1): 4-10.

13. Cernaro V, Santoro D, Lacquaniti A, Giuseppe Costantino, Luca Visconti, et al. (2016) Phosphate binders for the treatment of chronic kidney disease: Role of iron oxyhydroxide. Int J Nephrol Renovasc Dis 9: 11-19.

14. Yasuda G, Shibata K, Takizawa T, Ikeda Y, Tokita Y, et al. (2002) Prevalence of constipation in continuous ambulatory peritoneal dialysis patients and comparison with hemodialysis patients. Am J Kidney Dis 39(6): 1292-1299.

15. Lacquaniti A, Bolignano D, Campo S, Carlo Perrone, Valentina Donato, et al. (2009) Malnutrition in the elderly patient on dialysis. Ren Fail 31(3): 239-245.

16. Kalantar-Zadeh K, Block G, McAllister CJ, Humphreys MH, Kopple JD (2004) Appetite and inflammation, nutrition, anemia, and clinical outcome in hemodialysis patients. Am J Clin Nutr 80(2): 299-307.

17. Silva L, Lopes G, Matos C, Katherine Quadros Brito, Maurício Kauark Amoedo, et al. (2012) Gastrointestinal symptoms and nutritional status in women and men on maintenance hemodialysis. J Ren Nutr 22(3): $327-$ 335.

18. Lee SW, Song JH, Kim GA, Yang HJ, Lee KJ, et al. (2000) Effect of dialysis modalities on gastric myoelectrical activity in endstage renal disease patients. Am J Kidney Dis 36(3): 566-573.

19. Daniels G, Robinson J, Charles Walker, Jacquelyn S Pennings, Staci T Anderson (2015) Anderson Gastrointestinal symptoms among African Americans undergoing hemodialysis. Nephrol Nurs J 42(6): 539-548.

20. Bytzer P, Talley NJ, Leemon M, Young LJ, Jones MP, et al. (2001) Prevalence of gastrointestinal symptoms associated with diabetes mellitus: A population-based survey of 15,000 adults. Arch Intern Med 161(16): 1989-1996.

21. Fiderkiewicz B, Rydzewska-Rosołowska A, Myśliwiec M, Birecka M Kaczanowska B, et al. (2011) Factors associated with irritable bowel syndrome symptoms in hemodialysis patients. World J Gastroenterol 17(15): 1976-1981. 